중대뇌동맥에서 경두개색조이중도플러초음파와 경두개도플러초음파검사의 비교 연구

박정아 정근화 김정미 이우진 고상배 이승훈 윤병우

서울대학교 의과대학 서울대학교병원 신경과

\title{
Comparative Study of Transcranial Color-Coded Doppler and Transcranial Doppler Sonography in Middle Cerebral Artery
}

Jung-Ah Park, Keun-Hwa Jung, Jeong-Mi Kim, Woo-Jin Lee, Sang-Bae Ko, Seung-Hoon Lee, Byung-Woo Yoon

Department of Neurology, Seoul National University Hospital, Seoul National University College of Medicine, Seoul, Korea

Background: Transcranial color-coded Doppler (TCCD) is a real-time scanning method which evaluates intracranial vessels at high resolution. Whereas transcranial Doppler (TCD) cannot adjust the insonation angle between the ultrasonic wave and blood flow, TCCD can directly view the flow direction so that more accurate flow velocity can be derived. Meanwhile, it is necessary to compare the reference value of the parameters in each test.

Methods: Subjects with normal magnetic resonance angiography and TCD were enrolled in neurology clinics. Three test methods were applied for the measurement of middle cerebral arteries (MCAs): 1) Blind-TCD: Blind technique, 2) Image-TCCD: Image-based, and 3) AC-TCCD: angle corrected. Four sample points with depths of 65-48 mm were evaluated with indices including mean flow velocity (MFV), peak systolic velocity (Vs), end diastolic velocity (Vd), and pulsatility index (PI).

Results: Thirty patients with age of $61 \pm 17$ years were enrolled. MFVs at four depths of MCA were $58 \pm 10$ (Blind-TCD), $54 \pm 10$ (Image-TCCD), and $59 \pm 12 \mathrm{~cm} / \mathrm{s}$ (AC-TC$\mathrm{CD})$. There were no significant differences in the MFVs between Blind-TCD and AC-TCCD $(p=0.212)$. The Vs were $87 \pm 17$ (Blind-TCD), 90 \pm 17 (Image-TCCD) and $99 \pm 21 \mathrm{~cm} / \mathrm{sec}($ AC-TCCD) with significant differences $(p<0.05)$. The Vs increased in $14 \%$ at AC-TCCD over Blind-TCD. PI over age 65 in AC-TCCD was $20 \%$ higher than Blind-TCD.

Conclusion: Peak systolic velocity can be measured more accurately by AC-TCCD. Image-TCCD and AC-TCCD with small sample volumes are advantageous in that they reflect the change of depth-dependent blood flow more sensitively.

J Neurosonol Neuroimag 2018;10(1):25-33

Key Words: Transcranial color-coded Doppler; Transcranial Doppler; Angle correction; Middle cerebral artery
Received: October 27, 2017

Revised: May 10, 2018

Accepted: May 10, 2018

Address for correspondence: Keun-Hwa Jung

Department of Neurology, Seoul National University Hospital, 101 Daehak-ro, Jongno-gu, Seoul 03080, Korea Tel: +82-2-2072-4901 Fax: +82-2-3672-7553 E-mail:jungkh@gmail.com 


\section{JNN}

서 론

Doppler 원리를 이용한 초음파 진단법은 의학의 여러 분야 에서 적용되고 있으며, 특히 심장, 말초혈관, 경동맥 등의 혈류 를 평가하는 중요한 수단으로 이용되어 왔다. 그러나 두개골에 의하여 둘러싸여 있는 뇌혈관은 기존의 초음파가 투과될 수 없 어 진단에 한계가 있었다. 1982년 Aaslid에 의하여 처음 도입된 Tanscranial Doppler sonography (TCD)는 $2 \mathrm{MHz}$ 의 저주파 (low frequency, pulsed-waved mode)를 이용함으로써 두개 내 혈관의 혈류속도를 측정할 수 있게 되었으며, ${ }^{1}$ 현재는 신경 과에서 뇌혈관질환의 진단과 연구를 위한 필수적 장비로 자리 를 잡고 있다. 그러나 두개내 혈관의 혈류속도는 도플러 방정 식에 의한 혈관과 초음파의 입사각도 $\left(0-30^{\circ}\right.$ 이내 $)$ 가 매우 중 요한데, 혈관의 이미지를 보지 않고 검사하기 때문에 환자의 실제 혈관 구조와 초음파 입사각도를 맞추는 데에 있어 오차가 발생할 수 있다. TCD의 여러 선행 연구에서 중대뇌동맥의 주 행에는 개인차가 존재하고, probe의 위치나 투사각도(angle of insonation)를 정의할 수 없으므로 혈류속도가 불리하게 낮게 평가되거나 측정에 실패할 우려도 있다고 하였다. 2,3

서구에서는 이미 수십 년 전에 경동맥이중초음파 장비에 $2 \mathrm{MHz}$ probe 장착을 하여 실시간으로 고해상도(real time scanning, dynamic scanning, B-스캔) 영상을 이용하여 뇌혈관을 검사하고 있다. 이것이 경두개색조이중초음파검사 (transcranial color-coded duplex sonography, TCCD)이 다. TCCD는 두개내 혈관과 뇌실질 구조물을 고해상으로 이미 지화 시킨 검사법이다. $\mathrm{B}$-스캔으로 혈관의 해부학적 정보를 제공하므로 두개내 혈관의 정확한 동정이 가능하다. ${ }^{4}$ 뇌혈관의 혈류정보를 다가오는 혈관, 멀어지는 혈관으로 구분하여 칼라 도플러(color doppler)로 확인하고, 비정상적 측부순환과 선천 적 혈관 기형 및 저형성 등도 검출이 가능하다. 또한, 혈관 내 에서 원하는 곳에 도플러 용적(sample volume)을 위치시키기 가 가능하여 협착으로 인한 혈류속도 변화를 정밀하게 측정할 수 있으며, 혈관과 초음파의 투사각도를 시각적으로 정확하게 확인할 수 있다. ${ }^{5-7}$

기존의 $\mathrm{TCD}$ 검사는 간접적으로 얻은 혈류속도를 기준으로 평가(Blind-TCD)를 하였지만, 경두개색조이중초음파검사는 직접 혈관을 보면서 혈류속도와 혈관 구조를 관찰(Image-TC$\mathrm{CD})$ 하고, 초음파의 투사각도를 조절할 수 있어서 보다 정확한 혈류속도를 도출(angle corrected-TCCD, AC-TCCD)할 수 있다. 현재 임상 진료에서 가장 많이 사용 중인 Blind-TCD와 이의 단점을 보완할 수 있는 Image-TCCD, $\mathrm{AC}-\mathrm{TCCD}$ 는 향후 임상에서 함께 적용될 가능성이 높은 도구로 각 검사 수치의 의 미는 다를 수 있으므로 이에 대한 표준화가 필요하다. 우리나라 의 현재 TCCD 검사는 최근 일부 대학병원에서 시행되기 시작 하였으나, 아직 기준 참고수치에 대한 연구 및 $\mathrm{TCD}$ 검사 결과 와의 비교 연구는 시행된 바가 없다. 따라서, 이번 연구는 서울
대학교병원의 신경과 환자를 대상으로 병력, 임상 소견, 뇌 자 기공명혈관조영술(magnetic resonance angiography, MRA) 과 경두개도플러초음파 기준에 근거하여 정상군을 선정하여 두 개내 뇌혈관의 혈류속도에 대한 $\mathrm{TCD}$ 와 $\mathrm{TCCD}$ 의 정상 참고치와 검사에 따른 수치의 변화를 제시하고자 한다.

\section{대상 및 방법}

본 연구는 경두개도플러초음파검사(TCD)와 경두개색조이중 초음파(transcranial color-coded doppler, TCCD)에서 중 간 대뇌동맥(middle cerebral artery)의 혈류속도 차이를 비 교하는 단면적 연구로 서울대학교 생명윤리 심의위원회(Seoul National University Hospital Biomedical Research Institute)의 승인을 받아 수행하였다(H-1611-104-809). 2016 년 12월부터 2017년 5월까지 서울대병원 신경과 외래 진료를 본 환자 중 전문의에 의하여서 $\mathrm{TCD}$ 시행이 필요하다고 판단된 환자를 대상으로 하였다. TCD 검사 후 측정 뇌혈관의 혈류속 도가 정상이고 $\mathrm{MRA}$ 를 통하여 뇌혈관의 협착 및 변이가 없는 환자군을 대상으로 하였다.

연구 측정 방법은 세 가지 검사 방법으로 1) BlindTCD: blind technique, 2) Image-TCCD: image-based, 3) AC-TCCD: ultrasound beam과 vessel course가 맞지 않는 경우 angle correction 사용하여 검사한다(Fig. 1). 측정 혈관은 비교적 일관성 있게 검사가 가능한 중대뇌동맥(middle cerebral artery)으로 정하였다.

기존 발표된 해외 논문은 중대뇌동맥 깊이 $(65-48 \mathrm{~mm})$ 1개 의 sample을 측정하여서 평균 혈류속도(mean flow velocity) 를 분석하였지만, ${ }^{6}$ 본 연구에서는 Blind-TCD $\left(0-30^{\circ}\right)$, Im$\mathrm{age}-\mathrm{TCCD}\left(0^{\circ}\right), \mathrm{AC}-\mathrm{TCCD}\left(0-50^{\circ}\right)$ 검사에서 중대뇌동맥 깊이 $(65-48 \mathrm{~mm}) 4$ 개의 sample을 측정하여 비교하였다. 모 든 검사는 숙련된 검사자 한 명이 수행하였고, 결과의 분석은 두 명의 신경과 전문의에게 보고되어 판독되었다. 혈류속도 측 정변수와 인자는 평균 혈류속도(mean flow velocity, MFV), 수축기 혈류속도(peak systolic velocity [PSV]; Vs), 이완기 혈류속도(end diastolic velocity [EDV]; Vd), 박동성 지수 (pulsatility index, PI)를 포함하였다. 임상적으로 중대뇌동맥 의 $50 \%$ 협착의 진단기준은 TCD: MFV $>100 \mathrm{~cm} / \mathrm{s}$ 이상이며, TCCD: PSV $>155 \mathrm{~cm} / \mathrm{s}$ 이상, angle correction $>50^{\circ}$ 미만을 판독기준으로 제시한다. ${ }^{8}$

통계적 분석은 SPSS (ver. 22.0; IBM, Seoul, Korea)을 이용하여 모든 매개변수 간의 차이는 평균값과 표준편차로 기 록하였다. Blind-TCD, Image-TCCD, AC-TCCD 방법에서 얻은 측정치들의 평균치 비교는 Paired t-test와 ANOVA를 사용하였다. 결과의 통계적 검정은 $p$-value $<0.05$ 를 유의하 다고 판단하였다. 
A

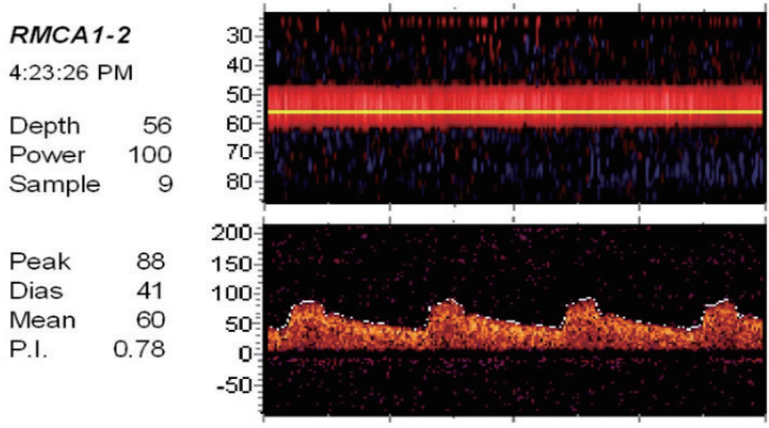

C

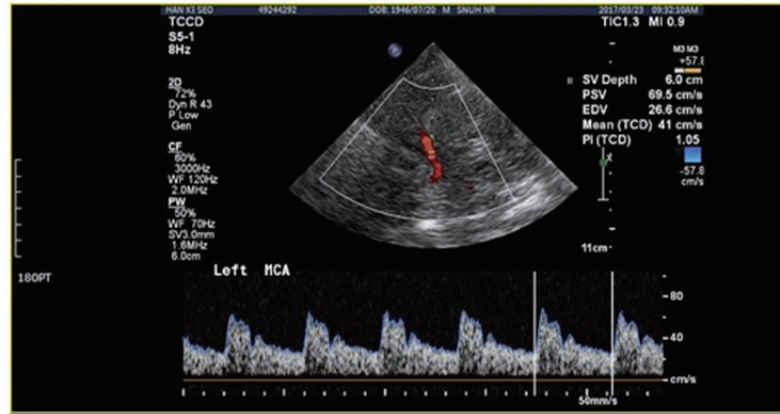

Angle correction $0^{\circ}$, MCA Mean: $41 \mathrm{~cm} / \mathrm{s}$, PI: 1.05
B

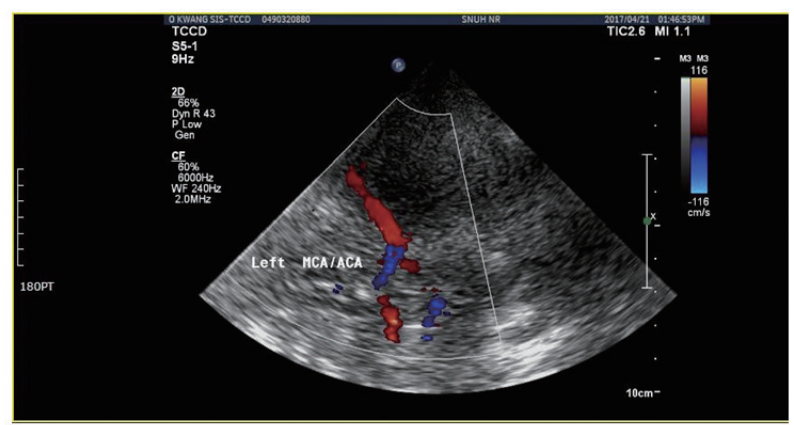

D

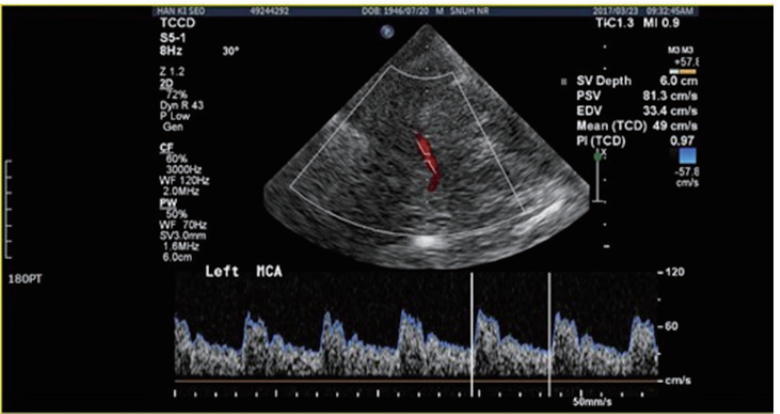

Angle correction $30^{\circ}$, Mean: $49 \mathrm{~cm} / \mathrm{s}, \mathrm{Pl}: 0.97$

FIG. 1. Methods of Blind-TCD, Image-TCCD, and AC-TCCD. (A) Doppler spectral signal by Blind-TCD. (B) 2D image by Image-TCCD, transtemporal approach, axial midbrain plane, middle cerebral artery (red), anterior cerebral artery (blue). (C, D) AC-TCCD, Upper: transcranial color duplex sonogram of the left middle cerebral artery (MCA). Lower: Doppler ultrasound waveform of the corresponding MCA toward flow pattern. The angle between the Doppler ultrasound beam (dotted line) and the course of the vessel (2 solid lines) is adjusted. Blind-TCD; blind-transcranial Doppler, Image-TCCD; image-based transcranial color-coded Doppler, AC-TCCD; angle corrected transcranial color-coded Doppler.

\section{결 과}

본 연구에 포함된 환자는 총 30명(남자 25명[83.3\%], 여

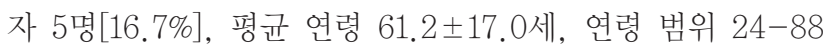
세)으로, 임상적 특성은 Table 1과 같다. 환자들의 중대뇌 동맥(middle cerebral artery, MCA)을 Blind-TCD, Image- $\mathrm{TCCD}, \mathrm{AC}-\mathrm{TCCD}$ 에서 동일 조건으로 4 개의 깊이 (depth)를 측정하여 각각 깊이에 따른 각각의 혈류속도 차이 를 비교하였다.

\section{1. $\mathrm{AC}-\mathrm{TCCD}$ 혈류속도 분석}

본 연구는 30 명 연구 대상자 개개인별로 2 개의 뇌 반구와 4 개 의 깊이에서 혈류속도를 측정하여 얻은 총 240 개 측정 자료를 대상으로 분석하였다. $\mathrm{AC}-\mathrm{TCCD}$ 검사에서 환자의 중대뇌동 맥의 주행 방향이 ultrasound beam과 vessel course가 맞지 않는 경우 angle correction을 시행하였다. Angle $<30^{\circ}$ 미만 에서는 MFV $55.57 \pm 14.52 \mathrm{~cm} / \mathrm{s}$ 이고, angle $31-40^{\circ}$ 에서는 MFV $75.00 \pm 15.25 \mathrm{~cm} / \mathrm{s}$, angle $41-50^{\circ}$ 에서는 MFV 71.00 $\pm 15.52 \mathrm{~cm} / \mathrm{s}$, angle $>50^{\circ}$ 이상에서 MFV $91.15 \pm 21.67 \mathrm{~cm} /$ $\mathrm{s}$ 였다. Angle값이 $30^{\circ}$ 이상으로 증가될수록 MFV가 점진적인
증가 경향의 결과를 보여주고 있으며, angle correction 방법 중에서 angle $>50^{\circ}$ 이상인 경우는 모두 20개였다. MCA 50\% 이상 협착(stenosis)의 판독기준으로 $\mathrm{MFV}>100 \mathrm{~cm} / \mathrm{s}$ 적용 하였을 때 angle $>50^{\circ}$ 이상되는 20 개의 수치 중 6 개가 $\mathrm{MCA}$ stenosis로 false positive result로 30\% 차지하였다(Fig. 2).

2. Blind-TCD, Image-TCCD, AC-TCCD 방법에 따른 혈류속도 parameter 분석

Blind-TCD, Image-TCCD, AC-TCCD에서 MCA의 혈 류속도 MVF, $\mathrm{Vs}, \mathrm{Vd}, \mathrm{PI}$ 들의 평균과 표준편차는 Table 2 와 같다. Blind- TCD와 $\mathrm{AC}-\mathrm{TCCD}$ 의 MFV는 유의한 차이 를 보이지 않았다 $(p=0.212)$. 반면, $\mathrm{Vs}$ 경우에는 Blind-TCD, Image-TCCD, AC-TCCD에서 유의한 차이를 보였는데, Blind-TCD < Image-TCCD < AC-TCCD 순으로 증가하는 경향을 보였고, $\mathrm{AC}-\mathrm{TCCD}$ 에서 Blind-TCD보다 약 $14 \%$ 증 가하였다(Fig. 3). Vd에서는 Image-TCCD에서 Blind-TCD, $\mathrm{AC}-\mathrm{TCCD}$ 보다 낮은 수치를 보였다. PI의 경우에는 Blind$\mathrm{TCD}$ 에 비하여 Image-TCCD, AC-TCCD에서 유의하게 증가 하는 경향을 보였다. 
TABLE 1. Study population characteristics

\begin{tabular}{lc}
\hline Variable & Value $(n=30)$ \\
\hline Age, years & $61.2 \pm 17.0$ \\
Male sex & $25(83.3)$ \\
Previous stroke history & $15(50)$ \\
Hypertension & $19(63.3)$ \\
Diabetes mellitus & $4(13.3)$ \\
Smoking in past 5 years & $5(16.7)$ \\
Coronary artery disease & $6(20)$ \\
Use of calcium channel blocker & $8(26.7)$ \\
Use of nitrates & $1(3.3)$ \\
\hline
\end{tabular}

Values are presented as number of patients (\%) or mean \pm standard deviation.

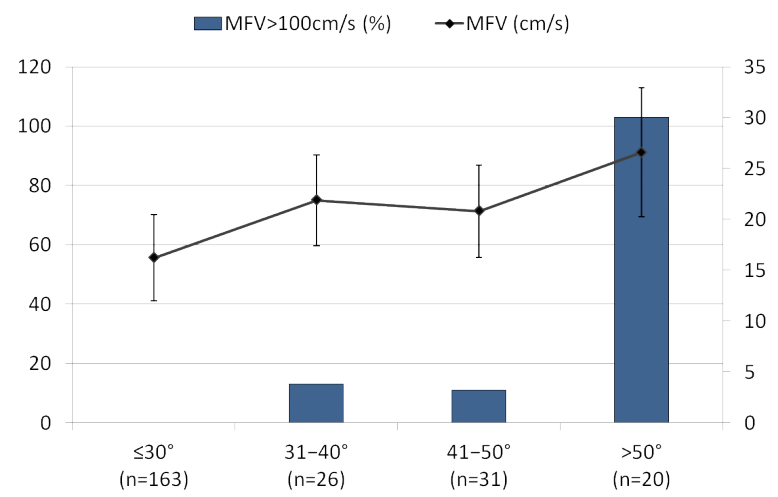

FIG. 2. Corrected angle-dependent mean flow velocity changes measured by TCCD. TCCD; transcranial color-coded Doppler, MFV; mean flow velocity.

TABLE 2. Comparisons of the blood flow parameters in middle cerebral artery across three methods

\begin{tabular}{|c|c|c|c|c|}
\hline Methods $(n=60)$ & $\mathrm{MFV}, \mathrm{cm} / \mathrm{s}$ & $\mathrm{Vs}, \mathrm{cm} / \mathrm{s}$ & $\mathrm{Vd}, \mathrm{cm} / \mathrm{s}$ & $\mathrm{PI}$ \\
\hline Blind-TCD & $58.03 \pm 10.72$ & $87.33 \pm 17.54$ & $39.82 \pm 8.643$ & $0.820 \pm 0.146$ \\
\hline Image-TCCD & $54.08 \pm 10.68$ & $90.54 \pm 17.54$ & $35.83 \pm 7.839$ & $1.035 \pm 0.302$ \\
\hline AC-TCCD & $59 \cdot 37 \pm 12.73$ & $99.70 \pm 21.97$ & $39 \cdot 34 \pm 9.483$ & $1.014 \pm 0.192$ \\
\hline$p$-value ${ }^{a}$ & $<0.001$ & 0.015 & $<0.001$ & $<0.001$ \\
\hline$p$-value ${ }^{b}$ & $<0.001$ & $<0.001$ & $<0.001$ & 0.514 \\
\hline$p$-value ${ }^{c}$ & 0.212 & $<0.001$ & 0.526 & $<0.001$ \\
\hline
\end{tabular}

Values are presented as mean \pm standard deviation.

MFV; mean flow velocity, Vs; peak systolic velocity, Vd; end diastoli c velocity, PI; pulsatility index, Blind-TCD; blind-transcranial Doppler, Image-TCCD; image-based transcranial color-coded Doppler, AC-TCCD; angle corrected-transcranial color-coded Doppler.

${ }^{a} p$-values comparing Blind-TCD and Image-TCCD.

${ }^{b} p$-values comparing Image-TCCD and AC-TCCD.

c $p$-values comparing AC-TCCD and Blind-TCD.

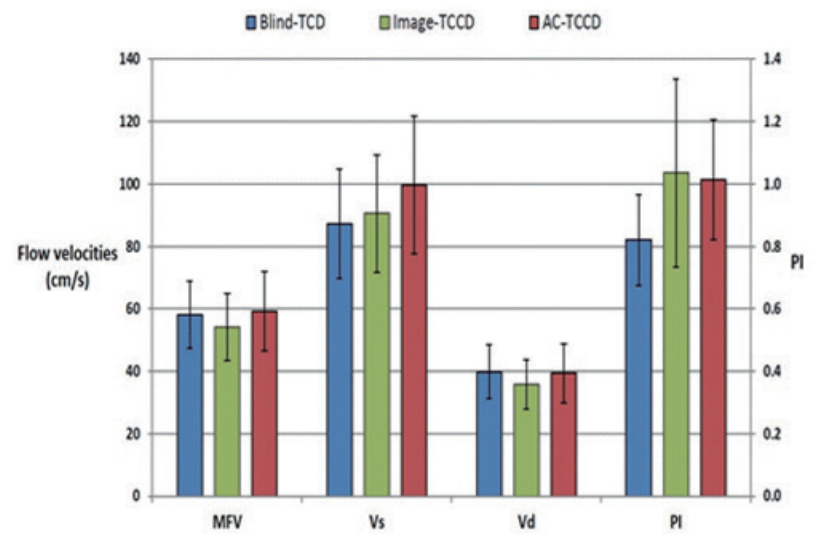

FIG. 3. Differences of sonographic parameters between Blind-TCD, Image-TCCD, and AC-TCCD. Blind-TCD; blind-transcranial Doppler, Image-TCCD; image-based transcranial color-coded Doppler, AC-TCCD; angle corrected transcranial color-coded Doppler, MFV; mean flow velocity, Vs; peak systolic velocity, Vd; end diastolic velocity, PI; pulsatility index.
3. Blind-TCD, Image-TCCD, AC-TCCD 방법에서 depth에 따른 혈류속도 분석

Blind-TCD, Image-TCCD, AC-TCCD에서 MCA의 4개 의 깊이 $60 \mathrm{~mm}, 56 \mathrm{~mm}, 50 \mathrm{~mm}, 48 \mathrm{~mm}$ 에 따른 혈류속 도 $\mathrm{MFV}, \mathrm{Vs}, \mathrm{Vd}, \mathrm{PI}$ 들의 각각 평균과 표준편차는 Table 3 과 같다. $\mathrm{MFV}$ 와 $\mathrm{Vd}$ 에서는 모든 depth에서 Blind-TCD가 Image-TCCD보다 높았고, AC-TCCD가 Image-TCCD보 다 높았다. 즉, Image-TCCD의 경우 모든 depth에서 가장 낮은 것으로 나타났다. $\mathrm{Vs}$ 의 경우에는 모든 depth에서 Image- TCCD와 $\mathrm{AC}-\mathrm{TCCD}$ 의 유의 수준은 $p<0.05$ 로 유의한 관계를 나타내었고, depth $56 \mathrm{~mm}$ 과 $50 \mathrm{~mm}$ 에서는 Blind$\mathrm{TCD}<$ Image-TCCD < AC-TCCD 순으로 높아지는 것으 로 나타났다. 특히, depth $56 \mathrm{~mm}$ 에서 AC-TCCD가 BlindTCD에 비하여 $20 \%$ 증가되어 있다(Fig. 4). PI의 경우에는 모 
든 depth에서 Blind-TCD와 Image-TCCD의 유의수준은 $p<0.01$ 이고, Blind-TCD와 $\mathrm{AC}-\mathrm{TCCD}$ 의 유의수준은 $p<0.01$ 로 유의한 관계를 나타내었다. Image-TCCD, $\mathrm{AC}-\mathrm{TCCD}$ 의 모든 depth에서 Blind-TCD보다 높은 수치값을 나타내었다. 그러나 Image-TCCD와 $\mathrm{AC}-\mathrm{TCCD}$ 간에는 유의한 차이가 관 찰되지 않았다.
4. Blind-TCD, Image-TCCD, AC-TCCD 방법에서 age 에 따른 혈류속도 parameter 분석

Blind-TCD, Image-TCCD, AC-TCCD에서 환자의 age 에 따른 $\mathrm{MCA}$ 의 $\mathrm{MFV}$ 와 $\mathrm{PI}$ 의 변화를 분석한 결과는 Table 4 에서 기술한다. Age 65세 이상군에서 65세 미만군에 비하 여 Blind-TCD, Image-TCCD, AC-TCCD에서 모두 통계 적으로 유의하게 $\mathrm{MFV}$ 가 낮았고 $\mathrm{PI}$ 가 높았다. Age를 중간값인 65세

TABLE 3. Comparisons of the blood flow parameters in several depths of middle cerebral artery across three methods

\begin{tabular}{|c|c|c|c|c|}
\hline & Depth $60 \mathrm{~mm}$ & Depth 56 mm & Depth $50 \mathrm{~mm}$ & Depth $48 \mathrm{~mm}$ \\
\hline \multicolumn{5}{|l|}{$\mathrm{MFV}, \mathrm{cm} / \mathrm{s}$} \\
\hline Blind-TCD & $59.75 \pm 12.03$ & $60.63 \pm 11.67$ & $57.58 \pm 11.65$ & $54.75 \pm 10.66$ \\
\hline Image-TCCD & $56.25 \pm 11.67$ & $58.78 \pm 14.07$ & $54 \cdot 35 \pm 12.35$ & $49.08 \pm 10.66$ \\
\hline AC-TCCD & $58.75 \pm 12.03$ & $66.23 \pm 19.53$ & $58.90 \pm 15.38$ & $53.60 \pm 15.48$ \\
\hline$p$-value ${ }^{a}$ & 0.004 & 0.066 & 0.007 & $<0.001$ \\
\hline$p$-value ${ }^{b}$ & 0.004 & $<0.001$ & $<0.001$ & 0.001 \\
\hline$p$-value & 0.489 & 0.004 & 0.416 & 0.472 \\
\hline \multicolumn{5}{|l|}{$\mathrm{Vs}, \mathrm{cm} / \mathrm{s}$} \\
\hline Blind-TCD & $90.16 \pm 19.19$ & $91.00 \pm 18.97$ & $86.35 \pm 18.98$ & $82.77 \pm 18.43$ \\
\hline Image-TCCD & $98.12 \pm 21.10$ & $98.19 \pm 24.25$ & $91.63 \pm 21.33$ & $82.65 \pm 22.36$ \\
\hline AC-TCCD & $98.12 \pm 11.00$ & $110.60 \pm 33.6$ & $99.91 \pm 25.60$ & $90.17 \pm 25.70$ \\
\hline$p$-value ${ }^{a}$ & 0.070 & $<0.001$ & 0.011 & 0.957 \\
\hline$p$-value ${ }^{b}$ & 0.004 & $<0.001$ & $<0.001$ & 0.001 \\
\hline$p$-value & 0.002 & $<0.001$ & $<0.001$ & 0.011 \\
\hline \multicolumn{5}{|l|}{$\mathrm{Vd}, \mathrm{cm} / \mathrm{s}$} \\
\hline Blind-TCD & $41.05 \pm 09.95$ & $41.71 \pm 09.70$ & $39.63 \pm 08.80$ & $37.50 \pm 08.02$ \\
\hline Image-TCCD & $37 \cdot 34 \pm 08.91$ & $39.02 \pm 10.37$ & $36.49 \pm 09.28$ & $31.79 \pm 09.89$ \\
\hline AC-TCCD & $38.98 \pm 09.13$ & $44.55 \pm 14.82$ & $39.22 \pm 11.56$ & $34.83 \pm 12.03$ \\
\hline$p$-value ${ }^{a}$ & $<0.001$ & 0.001 & 0.001 & $<0.001$ \\
\hline$p$-value ${ }^{b}$ & 0.004 & $<0.001$ & 0.001 & 0.001 \\
\hline$p$-value ${ }^{c}$ & 0.067 & 0.048 & 0.635 & 0.039 \\
\hline \multicolumn{5}{|l|}{$\mathrm{PI}$} \\
\hline Blind-TCD & $0.827 \pm 0.169$ & $0.816 \pm 0.167$ & $0.812 \pm 0.144$ & $0.823 \pm 0.166$ \\
\hline Image-TCCD & $0.997 \pm 0.222$ & $1.0153 \pm 0.227$ & $1.011 \pm 0.212$ & $1.016 \pm 0.210$ \\
\hline AC-TCCD & $0.998 \pm 0.218$ & $1.013 \pm 0.234$ & $1.029 \pm 0.212$ & $1.018 \pm 0.208$ \\
\hline$p$-value ${ }^{a}$ & $<0.001$ & $<0.001$ & $<0.001$ & $<0.001$ \\
\hline$p$-value ${ }^{b}$ & 0.848 & 0.840 & 0.053 & 0.797 \\
\hline$p$-value ${ }^{c}$ & $<0.001$ & $<0.001$ & $<0.001$ & $<0.001$ \\
\hline
\end{tabular}

Values are presented as mean \pm standard deviation.

MFV; mean flow velocity, Blind-TCD; blind-transcranial Doppler, Image-TCCD; image-based transcranial color-coded Doppler, AC-TC$\mathrm{CD}$; angle corrected transcranial color-coded Doppler, Vs; peak systolic velocity, Vd; end diastolic velocity, PI; pulsatility index.

${ }^{a}$-values comparing Blind-TCD and Image-TCCD.

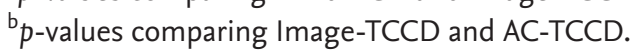

${ }^{c} p$-values comparing AC-TCCD and Blind-TCD. 

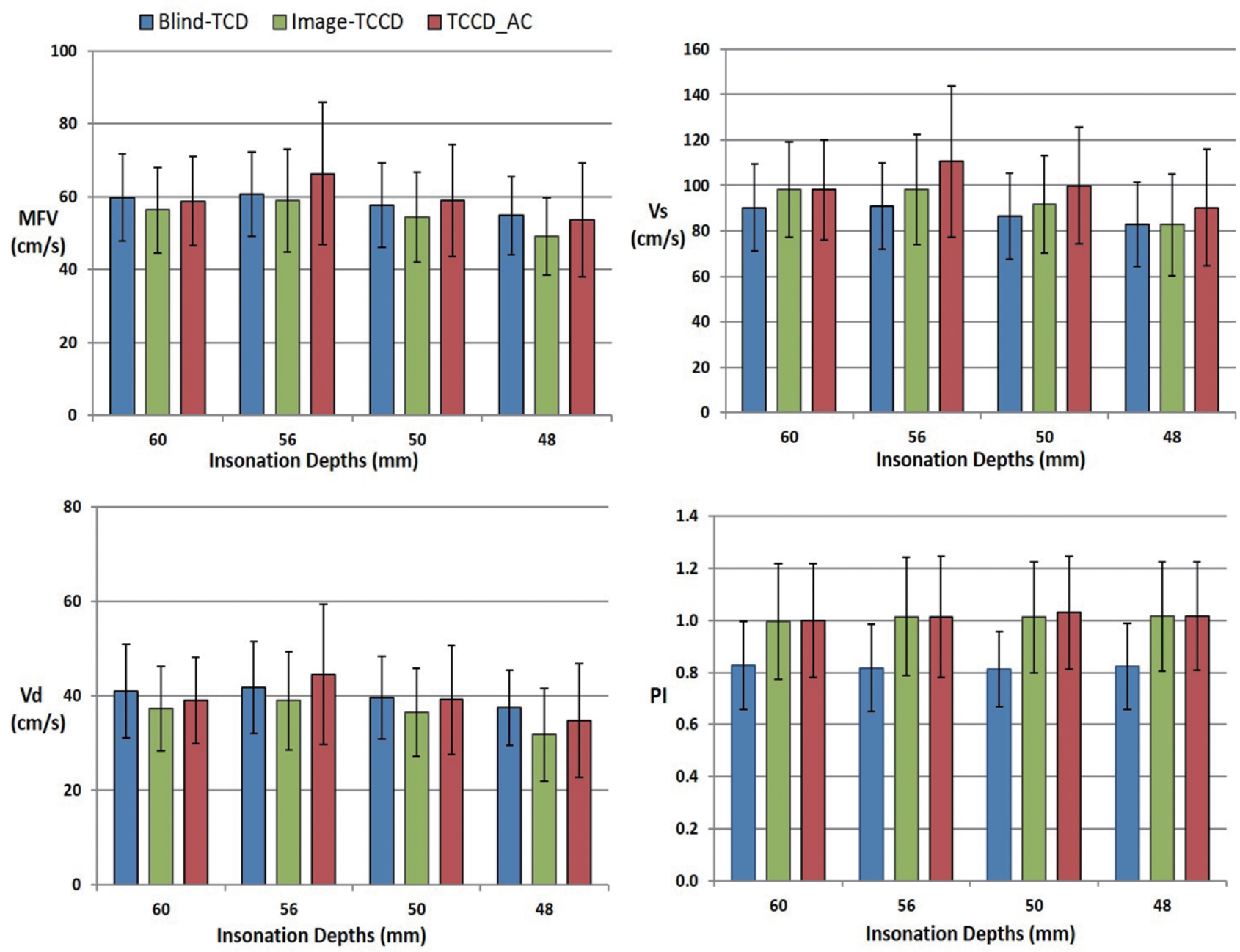

FIG. 4. Differences of sonographic parameters between Blind-TCD, Image-TCCD, and AC-TCCD according to sampling depth. Blind-TCD; blind-transcranial Doppler, Image-TCCD; image-based transcranial color-coded Doppler, AC-TCCD; angle corrected transcranial color-coded Doppler, MFV; mean flow velocity, Vs; peak systolic velocity, Vd; end diastolic velocity, Pl; pulsatility index.

TABLE 4. Differences of the blood flow parameters in middle cerebral artery according to age

\begin{tabular}{|c|c|c|c|c|c|c|}
\hline & \multicolumn{3}{|c|}{ MFV } & \multicolumn{3}{|c|}{$\mathrm{PI}$} \\
\hline & Blind-TCD & Image-TCCD & AC-TCCD & Blind-TCD & Image-TCCD & AC-TCCD \\
\hline \multicolumn{7}{|l|}{ Age, years } \\
\hline$<65(n=30)$ & $62.17 \pm 10.69$ & $57.58 \pm 11.27$ & $63.49 \pm 12.92$ & $0.765 \pm 0.142$ & $0.915 \pm 0.120$ & $0.923 \pm 0.121$ \\
\hline$\geq 65(n=30)$ & $53.88 \pm 9.175$ & $50.58 \pm 8.939$ & $55.25 \pm 11.29$ & $0.875 \pm 0.129$ & $1.155 \pm 0.375$ & $1.106 \pm 0.207$ \\
\hline$p$-value & 0.002 & 0.010 & 0.011 & 0.003 & 0.001 & $<0.001$ \\
\hline
\end{tabular}

Values are presented as mean \pm standard deviation.

MFV; mean flow velocity, PI; pulsatility index, Blind-TCD; blind-transcranial Doppler, Image-TCCD; image-based transcranial color-coded Doppler, AC-TCCD; angle corrected transcranial color-coded Doppler.

기준으로 하여 65 세 이상 $(\mathrm{n}=30)$ 과 65 세 미만 $(\mathrm{n}=30)$ 에서 각 검사 방법에 따른 측정 결과를 비교하였을 때 PI의 증가는 Blind-TCD에 비하여 Image-TCCD와 AC-TCCD에서 좀 더 뚜렷하였다(Fig. 5).
고 찰

건강인, 혈관연축(vasospasm), 혈관협착 환자에서 $\mathrm{TCCD}$ 의 연관성은 잘 알려져 왔고, angle correction에 따라서 혈 


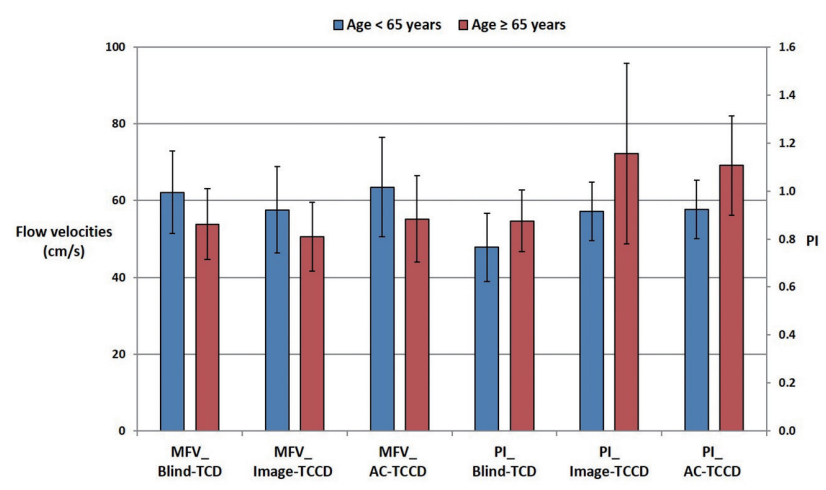

FIG. 5. Differences of sonographic parameters between Blind-TCD, Image-TCCD, and AC-TCCD according to age. Blind-TCD; blind-transcranial Doppler, Image-TCCD; image-based transcranial color-coded Doppler, AC-TCCD; angle corrected transcranial color-coded Doppler, MFV; mean flow velocity, PI; pulsatility index.

류가 증가되는 경향이 보고된 바 있다. ${ }^{8}$ 대다수의 선행 연구들 에서 angle correction은 TCCD의 하나의 장점이지만, angle correction의 사용의 범위가 매우 제한적이다. 이는 선천적 혈 관 변이(anatomic variation) 또는 개인에 따라 혈관의 굽어 짐(tortuosity) 또는 동일인에서 양쪽 차이(interhemispherically) 등으로 그 범위가 매우 가변적일 수 있다. 본 연구에서 도 개인에 따라 넓은 가변성 $\left(\right.$ angle $\left.0-70^{\circ}\right)$ 이 있음을 확인할 수 있었다. Hoksbergen 등 9 은 angle correction $>50^{\circ}$ 이상의 큰 각도에서는 높은 혈류속도를 도출하여 오류의 원인이 될 수 있기 때문에, 굽어짐 등에 구불구불한 혈관에서는 non angle correction을 권장하였다. 이번 연구에서도 angle correction $>50^{\circ}$ 이상의 큰 각도에서는 $\mathrm{MCA}$ 협착에 대한 false positive 도출할 가능성이 높음을 알 수 있었다. Angle이 $>50^{\circ}$ 연구 대 상자를 분석하여 보면 대부분 고령군에서 M1 혈관의 굽어짐이 심하고, M2에서는 혈관 굽어짐이 더 심하였다. 추후 고령군의 혈관 영상검사와 자세한 비교 연구가 필요하다고 생각된다.

$\mathrm{MCA}$ 에서 Blind-TCD, Image-TCCD, $\mathrm{AC}-\mathrm{TCCD}$ 의 평 균 혈류속도 parameter 분석에서는 $V_{S}$ 가 다른 변수들에 비 하여 높은 평균값을 보였다. Schöning 등의 보고에 의하면

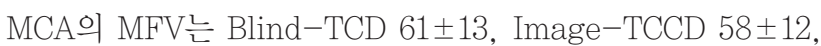
AC-TCCD $67 \pm 12(\mathrm{~cm} / \mathrm{s})$ 였다. Vs의 Blind-TCD $92 \pm 16$, Image-TCCD $92 \pm 18, \mathrm{AC}-\mathrm{TCCD} 107 \pm 18(\mathrm{~cm} / \mathrm{s})$ 였다. 즉, $\mathrm{AC}-\mathrm{TCCD}$ 방법에 의한 MFV, $\mathrm{VS}$ 가 약 10-15\% Blind-TCD 보다 높았다. ${ }^{10,11}$ 본 연구에서는 $\mathrm{MCA}$ 의 MFV는 Blind-TCD $58 \pm 10$, Image-TCCD $54 \pm 10$, AC-TCCD $59 \pm 12(\mathrm{~cm} / \mathrm{s})$ 로

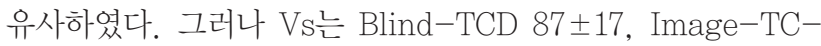
$\mathrm{CD} 90 \pm 17, \mathrm{AC}-\mathrm{TCCD} 99 \pm 21(\mathrm{~cm} / \mathrm{s})$ 로 Blind-TCD와 Image-TCCD에 비하여 $\mathrm{AC}-\mathrm{TCCD}$ 에서 $\mathrm{VS}$ 가 높았다. 본 연 구에서도 $\mathrm{AC}-\mathrm{TCCD}$ 에서 Blind-TCD보다 $\mathrm{Vs}$ 가 $14 \%$ 증가를 보였는데, 이것은 angle correction으로 인한 peak systolic velocity의 정확한 측정이 가능함 ${ }^{5}$ 을 보여주는 것이라고 사료 된다. 그러나 $\mathrm{MFV}$ 에서 Blind-TCD와 $\mathrm{AC}-\mathrm{TCCD}$ 의 유의수 준은 $p=0.212$ 로 유의하지 못하였다(Table 1). 이는 TCCD 검 사에서 $\mathrm{Vd}$ 의 저하 및 고령군에서 혈관탄성도의 변화가 영향을 주었을 가능성이 있다. ${ }^{12}$

$\mathrm{MCA}$ 에서 Blind-TCD, Image-TCCD, AC-TCCD의 4개 의 depth에 따른 혈류속도를 분석한 결과, Blind-TCD와 $\mathrm{AC}-\mathrm{TCCD}$ 에서 depth에 따른 $\mathrm{MFV}$ 와 $\mathrm{Vd}$ 의 측정값은 대체로 비슷하나, Image-TCCD에서는 MFV와 Vd값이 다른 두 측정 방법에 비하여 상대적으로 낮게 관찰되었다. 이는 소아 겸상적 혈구병 환자의 두개내 혈관에 대하여서 각 방법에 따른 $\mathrm{MFV}$ 를 비교한 선행 연구에서도 관찰되는 바이다. ${ }^{13}$ 해당 연구에서 $\mathrm{MFV}$ 의 값은 Image-TCCD를 통하여 측정하였을 때, Blind$\mathrm{TCD}$ 에 의한 측정에 비하여 평균 $20 \%$ 낮게 측정되었으며, 이 러한 차이는 내경동맥(internal carotid artery, ICA), 앞대뇌 동맥(anteriro cerebral artery, ACA), 후대뇌동맥(posterior cerebral artery, PCA) 등 insonation angle이 좋지 않은 혈 관에서 두드러졌고, Image-TCCD에 $\mathrm{AC}-\mathrm{TCCD}$ 을 시행함으 로써 상쇄되었다. 해당 연구에서 $\mathrm{MFV}$ 를 측정할 때 각 검사 방법별로 insonation depth가 최대 $20 \mathrm{~mm}$ 까지 차이가 났지 만 본 연구에서는 insonation depth를 통제한 점, 해당 연구 에서는 다양한 혈관에서 $\mathrm{MFV}$ 를 분석하였지만, 본 연구에서 는 $\mathrm{MCA}$ 만을 분석하였다는 점에 의하여 해당 연구의 결과와 본 연구에서 관찰된 바를 직접 비교하기는 무리가 있다. 하지 만 해당 연구와 본 연구에서 공통적으로 시사하는 바는, 특히 insonation angle이 좋지 않은 혈관에서 다양한 측정 오류가 발생할 수 있고, 이 경우 angle correction에 의하여 측정의 정확도가 향상될 수 있다는 점이다. ${ }^{13}$

한편, $\mathrm{Vs}$ 의 경우 Blind-TCD $<$ Image-TCCD $<\mathrm{AC}-\mathrm{TC}-$ $\mathrm{CD}$ 순으로 측정값이 증가하는 것으로 나타났으며, depth별 로 $56 \mathrm{~mm}$ 과 $50 \mathrm{~mm}$ 에서 좀 더 차이가 뚜렷하였다. Depth $56 \mathrm{~mm}$ 에서의 $\mathrm{Vs}$ 는 통계적인 분석에서도 Blind-TCD, Image-TCCD, $\mathrm{AC}-\mathrm{TCCD}$ 의 세 가지 측정 방법 모두에서 유 의한 결과를 보였다. 특히, depth $56 \mathrm{~mm}$ 에서 $\mathrm{AC}-\mathrm{TCCD}$ 가 Blind-TCD에 비하여 $20 \%$ 증가되었는데, 이것은 sample volume의 focality와 연관성이 있다고 사료된다. 이전 보고 된 depth에 따른 연구 분석이 없어서 본 연구와 비교를 할 수 없었다. 그러나 수축기 $(\mathrm{Vs})$ 지표의 증가가 Image-TCCD와 $\mathrm{AC}-\mathrm{TCCD}$ 가 Blind-TCD보다 더 높은 이유는 Image-TCCD 는 $\mathrm{MCA}$ 측정시 샘플 볼륨 $3 \mathrm{~mm}$ 적용하여서 검사하기 때문 이며, depth 간격을 작은 segment로 나눠서 검사가 가능하 여 Blind-TCD처럼 앞뒤 속도값에 영향이 없음을 확인하였다. 또한 Image-TCCD의 속도는 혈관을 $\mathrm{B}$-스캔 이미지와 색 도 플러(color doppler)에서 유동적인 혈류 흐름을 관찰하고(난혈 류 관찰 가능), 샘플 용적내 도플러 혈류속도를 측정하여 이중 점검(double check)이 가능하여 blind-TCD보다 정밀하게 
Jung-Ah Park, et al. Comparative Study of TCCD and TCD in MCA

측정할 수 있다. 즉, Image-TCCD, AC-TCCD는 높은 것은 높게 낮은 것은 낮게 측정 가능하여 국소 영역 측정이 가능하 다. 이것은 $\mathrm{Vs}$ 를 높게 잡을 수 있는 민감도와 연결지을 수 있 다.

선행 연구에 따르면 PI는 수축기, 이완기, 평균 혈류속도 사 이의 관계를 나타내며 혈관벽의 탄성(elasticity)과 관련이 있 다. 즉, $\mathrm{PI}$ 지표는 협착, 혹은 뇌압 항진을 의미하며 연령이 높 아짐에 따라 증가하는 경향이 있으며 age가 60세 이상에서 $\mathrm{MFV}$ 는 감소하고 $\mathrm{PI}$ 는 증가된다 ${ }^{14,15}$ 고 보고가 있다. 본 연구 에서도 Blind-TCD < Image-TCCD < AC-TCCD에서 age 65세 이상에서 65 세 미만에 비하여 $\mathrm{PI}$ 는 증가하였고, $\mathrm{MFV}$ 는 감소하는 것으로 관찰되었다. 특히, $\mathrm{PI}$ 의 경우 65 세 이상 $\mathrm{AC}-\mathrm{TCCD}$ 에서 Blind-TCD보다 20\% 높게 측정되었다. 이 와 같은 결과도 angle correction을 사용하여 혈관의 빠른 유 속 흐름을 반영한 선행 연구의 결과를 뒷받침하는 것으로 사 료된다. ${ }^{10}$ 또한, 고령의 나이에서 PI의 증가는 Image-TCCD, $\mathrm{AC}-\mathrm{TCCD}$ 에서 특히 높음을 보여주었는데, 이는 $\mathrm{Vs}$ 증가와 sample volume의 focality와 연관성이 있다고 사료된다. 향 후 고령군 대상으로 PI 비교를 위한 후속 연구 연구가 필요할 것으로 생각된다.

본 연구에서는 몇 가지 제한점을 가지고 있다. 첫째, 30 명 의 적은 연구 대상자 수의 한계로 혈관 위험인자 및 기타 혈관 질환들과의 관련성을 알아보는 데는 어려움이 있었다. 둘째, 이번 연구는 1명의 검사자에 의하여 수행된 결과로서 Blind$\mathrm{TCD}$, Image-TCCD, $\mathrm{AC}-\mathrm{TCCD}$ 에 측정에 있어 다른 검사자 간에 재현성(reproducibility) 검증을 하여 측정치의 정량적인 관계를 알아보는 데는 무리가 있었다. 셋째, angle correction 으로 $\mathrm{MFV}$ 가 많이 증가된 환자에 대하여서는 $\mathrm{MRI} / \mathrm{MRA}$ 와 의 비교도 가능할 것으로 판단하나 sample 수가 작고 $\mathrm{im}-$ age-TCD와 MRA 시행 간격이 다양한 것이 제한적이었다. 넷 째, 검사자의 기술적(technical) 측면으로 angle correction은 $\mathrm{MCA}$ 의 주행 방향에 맞추어 정확한 속도 반영함을 위함인데, 혈관이 구불구불한 경우 트랜스듀서를 조작하여 혈관 동정하 는 것에 매우 어려움이 있었다. 또한 대뇌혈관의 직경이 2-4 $\mathrm{mm}$ 로 작기 때문에 색 도플러만 보이고 혈관벽은 잘 가시화되 지 않는다는 점도 제한점이었다. 따라서 검사 방법의 신뢰성 있는 angle with correction 방법의 표준화는 매우 어려움이 따를 것으로 판단된다. 또한 $\mathrm{ACA}, \mathrm{PCA}$ 등 혈관 주행에 굴곡 이 있는 다른 intracranial artery에 angle correction 적용 을 획일적으로 표준화할 수 없다는 것 또한 제한점이었다. 이 것은 병원 간, 검사자 간 동일 검사를 추적 관찰시에 이전 검 사의 angle 재현성에 문제 있다. 추후에 이러한 문제점을 보안 하여 더욱 객관화한다면 뇌혈관 질환자들의 혈류역학적 평가 에 추적 검사 및 치료 효과를 판단하는데 많은 도움을 줄 수 있 을 것으로 기대된다.

\section{REFERENCES}

1. Aaslid R, Markwalder TM, Nornes H. Noninvasive transcranial Doppler ultrasound recording of flow velocity in basal cerebral arteries. J Neurosurg. 1982;57:769-774.

2. Aaslid R. The Doppler principle applied to measurement of blood flow velocity in cerebral arteries. In: Aaslid R. Transcranial doppler sonography. Vienna: Springer. 1986;22-38.

3. Alexandrov AV, Sloan MA, Wong LK, Douville C, Razumovsky AY, Koroshetz WJ, et al. Practice standards for transcranial Doppler ultrasound: part I--test performance. J Neuroimaging. 2007;17:11-18.

4. Martin PJ, Evans DH, Naylor AR. Measurement of blood flow velocity in the basal cerebral circulation: advantages of transcranial color-coded sonography over conventional transcranial Doppler. J Clin Ultrasound. 1995;23:21-26.

5. Krejza J, Mariak Z, Walecki J, Szydlik P, Lewko J, Ustymowicz A. Transcranial color Doppler sonography of basal cerebral arteries in 182 healthy subjects: age and sex variability and normal reference values for blood flow parameters. AJR Am J Roentgenol. 1999;172:213-218.

6. Bogdahn U, Becker G, Winkler J, Greiner K, Perez J, Meurers B. Transcranial color-coded real-time sonography in adults. Stroke. 1990;21:1680-1688.

7. Brouwers PJ, Vriens EM, Musbach M, Wieneke GH, van Huffelen AC. Transcranial pulsed Doppler measurements of blood flow velocity in the middle cerebral artery: reference values at rest and during hyperventilation in healthy children and adolescents in relation to age and sex. Ultrasound Med Biol. 1990;16:1-8.

8. Baumgartner RW, Mattle HP, Schroth G. Assessment of $\geq 50 \%$ and $<50 \%$ intracranial stenoses by transcranial color-coded duplex sonography. Stroke. 1999;30:87-92.

9. Hoksbergen AW, Legemate DA, Ubbink DT, Jacobs MJ. Success rate of transcranial color-coded duplex ultrasonography in visualizing the basal cerebral arteries in vascular patients over 6o years of age. Stroke. 1999;30:1450-1455.

10. Krejza J, Mariak Z, Babikian VL. Importance of angle correction in the measurement of blood flow velocity with transcranial Doppler sonography. AJNR Am J Neuroradiol. 2001;22:1743-1747.

11. Schöning M, Buchholz R, Walter J. Comparative study of transcranial color duplex sonography and transcranial Doppler sonography in adults. J Neurosurg. 1993;78:776-784.

12. Ackerstaff RG, Keunen RW, van Pelt W, Montauban van Swijndregt AD, Stijnen T. Influence of biological factors on 
changes in mean cerebral blood flow velocity in normal ageing: a transcranial Doppler study. Neurol Res. 1990;12:187191.

13. Krejza J, Rudzinski W, Pawlak MA, Tomaszewski M, Ichord $\mathrm{R}$, Kwiatkowski J, et al. Angle-corrected imaging transcranial Doppler sonography versus imaging and nonimaging transcranial doppler sonography in children with sickle cell disease. AJNR Am J Neuroradiol. 2007;28:1613-1618.
14. Grolimund P, Seiler RW. Age dependence of the flow velocity in the basal cerebral arteries--a transcranial Doppler ultrasound study. Ultrasound Med Biol. 1988;14:191-198.

15. de Riva N, Budohoski KP, Smielewski P, Kasprowicz M, Zweifel C, Steiner LA, et al. Transcranial Doppler pulsatility index: what it is and what it isn't. Neurocrit Care. 2012;17:5866. 